Artículo Original

\title{
Conductas de autoexamen cutáneo en dermatólogos chilenos
}

\author{
Perla Calderón ${ }^{1}$, Mauricio Villagra ${ }^{2}$, Juliana Bedoya Martin³, Catalina Bley ${ }^{4}$, Isidora Harz ${ }^{5}$, Miguel Espinoza ${ }^{1}$
}

\section{Resumen}

La incidencia de cáncer de piel ha aumentado, por lo que la detección precoz puede ser beneficiosa. Se ha sugerido que los dermatólogos son una población de alto riesgo para desarrollar cáncer de piel. El objetivo de este estudio es evaluar la frecuencia de autoexamen, consulta dermatológica y cáncer de piel en un grupo de dermatólogos chilenos.

Pacientes y métodos: Se confeccionó una encuesta de 8 preguntas, hecha personalmente, durante 4 meses, a 143 dermatólogos de la Sociedad Chilena de Dermatología (46,7\%). Las variables analizadas fueron: sexo, edad, fototipo de Fitzpatrick, frecuencia de autoexamen, consulta a otro dermatólogo, antecedente de melanoma maligno (MM), carcinoma basocelular $(\mathrm{CBC})$, carcinoma espinocelular (CEG) y queratosis actínica (QA), y razones para no realizarse examen cutáneo en forma regular.

Resultados: El 60\% de encuestados se hacía autoexamen, pero 48\% afirmó hacerlo sólo ocasionalmente. Con respecto a si solicitan ser evaluados por otro dermatólogo, el 81\% no lo hace, del 19\% que si lo hace, un 69\% consultaba sólo ante una lesión llamativa. Finalmente, el 17\% de encuestados refirió haber tenido neoplasias cutáneas. Entre ellas 12\% para QA, 5\% para $\mathrm{CBC}, 2 \%$ para CEC y ningún melanoma.

Conclusión: La mayoría de los dermatólogos encuestados se realiza autoexamen, pero no regularmente, y raramente hacen consultas de chequeo a sus colegas, la mayoría solicita evaluación por otro dermatólogo ante una lesión sospechosa.

Palabras clave: Autoexamen cutáneo; autocuidado médico; prevención de cáncer de piel.

\section{$\mathrm{L}$} a tendencia actual de la incidencia de cáncer de piel en la Región Metropolitana, en Chile y el mundo es al alza. ${ }^{1,6}$ Su detección precoz per-

${ }^{1}$ Servicio de Dermatología, Hospital Clínico Universidad de Chile. Santiago, Chile. ${ }^{2}$ Interno de Medicina, Facultad de Medicina Universidad de Chile. Santiago, Chile. ${ }^{3}$ Fundación Arturo López Pérez. ${ }^{4} \mathrm{H}$ ospital de Rancagua. ${ }^{5}$ Residente de Dermatología Pontificia Universidad Católica

Correspondencia: Perla Calderón Herschman. Correo electrónico: perlacald@yahoo.com. Celular: +56 229788173. Dirección: Santos Dumont 999, Independencia. Código postal: 8380456.

\section{SUMMARY}

Abstract: Skin cancer incidence has increased worldwide; early detection may be beneficial. Some evidence suggests dermatologists as a high-risk population for developing skin cancer. The aim of this study was to assess how often Chilean dermatologists consult with colleagues for check ups, to evaluate the frequency of self-skin examination and the frequency of skin cancer in this group.

Patients and methods: A sample of 143 members (46,7\%) of the Chilean Society of Dermatology responded an eight items questionnaire, in a face-to-face manner. Evaluated variables were: sex, age, Fitzpatrick phototype, self-skin examination frequency, frequency for consult s with another dermatologist, previous history of malignant melanoma, basal cell carcinoma, squamous cell carcinoma and actinic keratosis, and reasons to perform or not in themselves regular skin examination.

Results: $60 \%$ performed regular self-skin examination, but only $48 \%$ did it regularly. Concerning whether they consulted another dermatologist, $81 \%$ didn't, and between the $19 \%$ who do consult, $69 \%$ of them consulted only in case of a suspicious lesion. Finally, $17 \%$ of the surveyed people referred history of skin cancer. $12 \%$ were actinic keratosis, 5\% basal cell carcinoma, $2 \%$ squamous cell carcinoma and no melanoma was declared.

Conclusion: Most of surveyed dermatologists perform occasional self-skin examination, and rarely do regular check ups with colleagues, most of them consulted another dermatologist only if a suspicious lesion was found.

Key words: Cutaneous self examination; doctors selfcare; skin cancer prevention.

mite disminuir la morbimortalidad, impacto psicosocial y disminuye costos de tratamiento. ${ }^{7}$ En este contexto el autoexamen es importante, sin embargo, la prevalencia de esta práctica es generalmente baja, incluso en poblaciones de alto riesgo ${ }^{8}$, dentro de las cuales podrían estar los dermatólogos o tal vez los médicos en general. ${ }^{9}$

El examen de la superficie total de la piel puede aumentar hasta seis veces la probabilidad de detectar el melanoma comparado con el examen parcial. Los hombres tienen más lesiones en la espalda y las mujeres en las piernas, por lo que un examen en estas áreas podría par- 
ticularmente ayudar en la detección temprana. ${ }^{10}$ Saitta et al, concluyeron que los dermatólogos tienden más a realizarse un autoexamen en lugar de visitar a otro dermatólogo para un examen total de la piel. ${ }^{11}$ Las razones para esta conducta de riesgo, no han sido estudiadas, pero podrían tener que ver con conductas habitualmente negligentes de los médicos ante su propia salud, ${ }^{12}$ no querer importunar a colegas, negación y otras aún no aclaradas.

\section{Objetivos}

El objetivo de este estudio es evaluar la frecuencia de autoexamen cutáneo, consulta dermatológica regular para chequeos, y cáncer de piel en dermatólogos chilenos. Un objetivo secundario es crear conciencia entre los dermatólogos lo cual permitiría generar cambios de conducta que aseguren una detección temprana de cáncer de piel tal cual se les recomienda a los pacientes habitualmente.

\section{MÉtodo}

Diseño y participantes: Se confeccionó una encuesta de ocho preguntas de alternativas que se aplicó a 143 dermatólogos pertenecientes a la Sociedad Chilena de Dermatología (SOCHIDERM), con modalidad cara a cara, por residentes de dermatología.

Encuesta: Se analizó las siguientes variables: sexo, edad, fototipo de Fitzpatrick, antecedente personal de cáncer de piel y la frecuencia de autoexamen cutáneo y chequeo cutáneo realizado por otro dermatólogo, y razones para no realizarse examen cutáneo en forma regular en caso de no hacerlo. La encuesta fue validada previamente en el Servicio de Dermatología del Hospital Clínico de la Universidad de Chile.

Recolección de información: De forma presencial se aplicó la encuesta a los participantes en su lugar de trabajo o durante reuniones científicas, en un período de 4 meses en 2013. Los participantes fueron voluntarios y no se ofreció incentivos para completar las encuestas.

\section{Resultados}

Se encuestaron en total 143 dermatólogos pertenecientes a la SOCHIDERM correspondientes al 46,7\% de sus integrantes. De ellos el $65 \%$ eran sexo femenino y el $35 \%$ masculino.

La distribución por rangos de edad fue de 16\% para el rango entre 25-35 años, 32,9\% para el rango entre 36-45 años, 25,2\% para el rango entre 46-55 años, 19,6\% para el rango entre 56-65 años, 5,6\% para el rango entre 6675 años y $0,7 \%$ para los mayores de 75 años. (Grafico 1)

El 55,2\% de los encuestados señaló tener fototipo III de Fitzpatrick.

En relación al antecedente de cáncer de piel el 83,2\% de los encuestados señaló no haber tenido nunca una lesión cutánea maligna o pre-maligna, del resto el 11,9\% refirió haber tenido queratosis actínicas, el 5\% carcinoma basocelular y el $2 \%$ carcinoma espinocelular. Ningún dermatólogo declaró historia personal de melanoma.

El $60 \%$ de los encuestados manifestó realizarse autoexamen cutáneo, de los cuales un 48,2\% afirmó hacerlo sólo ocasionalmente. (Gráfico 2).

Con respecto al control cutáneo realizado por otro dermatólogo el $18,9 \%$ respondió afirmativamente, sin embargo, de ellos el 69,2\% consultaba sólo ante una lesión lla-

mativa. (Gráfico 3).

Las principales razones para no tener un control regular fueron: "falta de tiempo" (24,5\%), "no molestar a otro colega" (18,2\%), "pudor" (6,9\%) y "otras" (50,3\%).

\section{Discusión}

La literatura sugiere que los dermatólogos podrían ser un grupo de alto riesgo para cáncer de piel, por nivel 


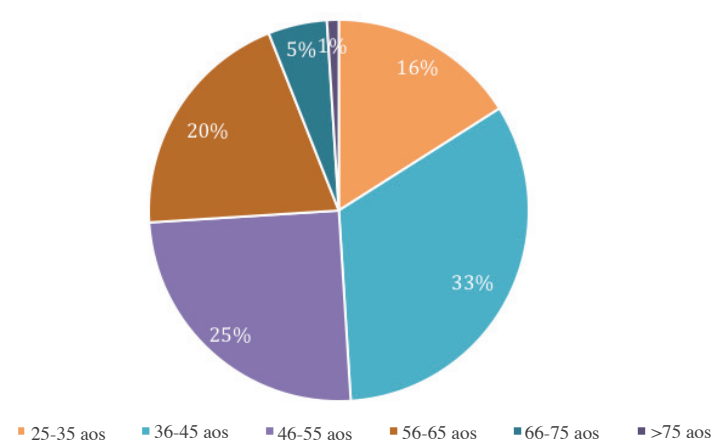

Gráfico $N^{0} 1$

Porcentajes por grupo etario de dermatólogos encuestados

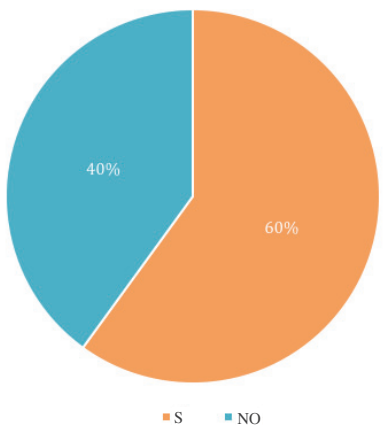

Gráfico $\mathrm{N}^{\boldsymbol{o}} \mathbf{2}$

Porcentaje de dermatólogos encuestados que se realizan autoexamen

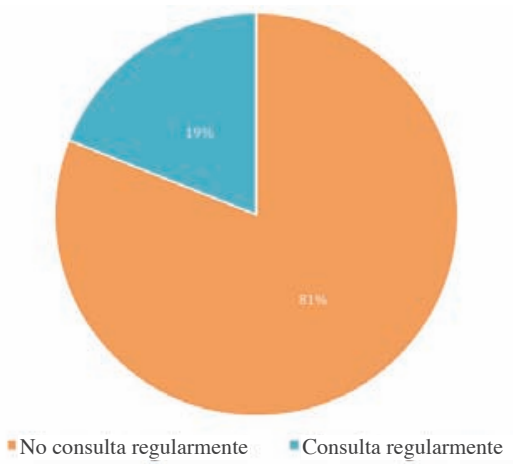

Gráfico $N^{\boldsymbol{o}} 3$

Porcentaje de encuestados que consultan con otro dermatólogo regularmente

educacional, nivel socioeconómico y hábitos vacacionales, entre otros, ${ }^{13,27}$ lo que deberá ser mejor investigado, es posible que eso sea extrapolable a todos los médicos, que suelen tener una conducta descuidada frente a la propia salud.

En nuestro estudio, el 60\% de los encuestados manifestó hacerse autoexamen cutáneo periódico, algo menor a lo obtenido por Saitta (71\%), sin embargo, esta cifra parece bajo lo esperable, considerando que un $40 \%$ declara nunca hacerse un autoexamen y contrasta con lo que se aconseja a los pacientes. Es posible que el hecho de haber realizado la encuesta "cara a cara", pueda haber aumentado falsamente esta cifra. Se desconoce información que permita comparar este valor con el de la población general en Chile que se realiza autoexamen o examen con dermatólogo.

Solo el 18,9\% consulta a colegas para examen cutáneo, pero de ellos la mayoría lo hace solo ante una lesión sospechosa. Las razones más citadas en nuestra encuesta fueron falta de tiempo $(24,5 \%)$ y no molestar a colegas $(18,2 \%)$, entre otras.
Creemos que debe incorporarse instancias de reflexión sobre autocuidado, que nos permitan lidiar con la sensación de falta de tiempo, y trabajar en la confianza necesaria para realizarse exámenes regularmente sin que exista la idea que se molesta al colega. Si no tenemos conductas apropiadas de autocuidado, no es razonable pedir eso de nuestros pacientes.

Si bien el autoexamen es una herramienta que ha demostrado ser valiosa, ${ }^{28}$ no es suficiente en algunas áreas de difícil visualización tales como dorso, cuero cabelludo, orejas y mucosas. Además, hoy en día es fundamental el uso del dermatoscopio para evaluar lesiones cutáneas, y para ello es indispensable contar con el examen de un colega. El 81\% de los encuestados no consulta regularmente a colegas para ser examinado por completo, este alto número puede constituir una conducta de riesgo que impida la detección precoz de cáncer de piel.

En cuanto a los antecedentes de cáncer de piel en los encuestados, resultaron muy similares, de $17 \%$ en nuestro trabajo y el de Saitta et al. Eso significa que casi 1 de cada 5 dermatólogos podría tener una lesión neoplásica 
cutánea a lo largo de la vida. Este valor es importante y debería ayudar a crear conciencia sobre la importancia de practicar lo que se predica.

Dentro de las limitaciones de este estudio está el número de participantes que, aunque significativo, para el universo de dermatólogos, deja afuera al 54\%. Sin embargo, fue mucho más alto que el $16 \%$ de respuesta reportado en el trabajo de Saitta, el cual fue realizado vía correo electrónico. El hecho que la encuesta fue realizada de manera presencial pudo haber disminuido el número de lesiones reportadas por los dermatólogos encuestados, ya que podría ser información manejada como confidencial por muchos.

Este es el primer estudio de este tipo en Chile, y de los pocos en el mundo, hasta donde se tiene conocimiento.

Nuevas investigaciones deberían profundizar en las razones para no hacer chequeos preventivos con regularidad. Las sociedades médicas deberían promover el autocuidado de los médicos regularmente y evaluar formas de facilitarles el acceso a estas prácticas.

\section{Conclusiones}

La mayoría de los dermatólogos chilenos encuestados se realiza autoexamen cutáneo, pero no regularmente y sólo solicita evaluación por otro dermatólogo ante una lesión sospechosa.

La mayoría de los encuestados no consulta regularmente a colegas para examen cutáneo total, es indispensable crear mayor conciencia sobre esta falencia que constituyen un factor de riesgo en detección precoz de cáncer de piel.

Existen limitaciones para consultar que debieran ser analizadas y abordadas por las sociedades de dermatología, con el fin de que se tome conciencia de la importancia del autocuidado en salud y se haga más fácil el acceso a colegas para examen cutáneo preventivo al igual que se les aconseja a los pacientes.

\section{REFERENCIAS BIBLIOGRÁFICAS}

1. Siegel RL, Miller KD, Jemal A. Cancer statistics, CA Cancer J Clin 2016; 66:7

2. Zemelman V, Roa J, Díaz G, Araya I, Zamalloa G, Faúndez E. Aumento de la incidencia del cáncer cutáneo en hospitales públicos de la Región Metropolitana (1992-1998). Dermatol. Chile 2001; $17: 180-5$

3. Zemelman V, Garmendia ML, Kirschbaum A: Malignant Melanoma Mortality in Chile (1988-98). Int J Dermatol 2002, 41:99-103

4. Riquelme J, Zemelman V, Paredes D, Araya MC, Durán V. Análisis de variables epidemiológicas, clínicas, e histopatológicas del cáncer de piel en el hospital regional de Arica ( 2007-2011) Rev Chilena Dermatol 2014; 30(3):279-284

5. Schrag B, Zemelman V, Yagnam M. Estudio epidemiológico de cáncer de piel del Hospital Regional de Iquique, Región de Tarapacá de Chile (2006-2010). Rev Chilena Dermatol 2012; 28:280-286

6. Zemelman V, Silva C, Tamblay N. "Tasas de incidencia y caracterización de tumores cutáneos en el Servicio de Salud Aconcagua (1999- 2001)" Rev Chilena Dermatol 2002; 18(4):281284

7. Kopf A, Salopek T, Slade J, Marghoob A, Bart R. Techniques of cutaneous examination for the detection of skin cancer. Cancer 1995; 75:684-690

8. Aitken JF, Janda M, Lowe JB, Elwood M, Ring IT, youl PH, et al. Prevalence of whole-body skin self-examination in a population at high risk for skin cancer (Australia). Cancer Causes Control 2004; $15: 453$

9. Marghoob A, Slade J, Salopek T, Kopf AQ, Bart RS, Rigel DS. Basal cell and squamous cell carcinomas are important risk factors for cutaneous malignant melanoma. Cancer 1995; 75(Supp):707-714

10. Langley RG, Fitzpatrick TB. Clinical characteristics. En: Balch CM, Houghton AN, Sober AJ, Soong SJ (Editores). Cutaneous melanoma, Quality Medical Publishing, Inc, St. Louis 1998. p.81

11. Saitta P, Cohen DE, Rigel D, Grekin SK, Brancaccio R. The frequency of Self-skin examination and full body skin examination in dermatologists. J Clin Aesthet Dermatol 2011; 4(6):20-24

12. Blackwelder R, Watson KH, Freedy J. Physician Wellness Across the Professional Spectrum. Prim care 2016 Jun; 43(2):355-61

13. Ries LAG, Harkins D, Krapcho M, et al (eds). SEER Cancer Statistics Review, 1975- 2003. Bethesda, MD: National Cancer Institute. http://seer.cancer.gov/csr/1975_2003/. Based on November 2005 SEER data submission, posted to the SEER website, 2006

14. Pion I, Rigel D, Garfinkel L, Silverman M, Kopt AW. Occupation and the risk of malignant melanoma. Cancer 1995; 707-714

15. Williams RR, Horn JW. Association of cancer sites with tobacco and alcohol consumption and socioeconomic status of patients: interview study from the Third National Cancer Survey. J Natl Cancer Inst 1977; 59:1147-1185

16. Graham S, Marshall J, Haughey B. An inquiry into the epidemiology of melanoma. Am J Epidemiol 1985; 122:606-619.31 
17. Gallagher RP, Elwood JM, Threlfall WJ, Spinelli JJ, Fincham S, Hill GB. Socioeconomic status, sunlight exposure and risk of malignant melanoma: the Western Canada Melanoma Study. J Natl Cancer Inst 1990; 46:1-4.32

18. Kirkpatrick CS, Lee JAH, Emily W. Melanoma risk by age and socioeconomic status. Int J Cancer 1990; 46:1-4

19. Lee PY, Silverman MK, Rigel DS. Level of education and 33. the risk of malignant melanoma. J Am Acad Dermatol 1992; 26:59-63.34

20. Krain LS. Malignant melanoma of the skin in California, 1942-1969: epidemiologic and survival considerations. Cutis 1973; 11:350-357

21. Eklund G, Malec E. Sunlight and the incidence of cutaneous 35. malignant melanoma: effect of latitude and domicile in Sweden. Scand J Plast Reconstr Surg 1978; 12:231-241. 36

22. Lee JAH, Strickland D. Malignant melanoma, social status and outdoor work. Br J Cancer 1980; 41:757-763.37

23. Cooke KR, Skegg DCG, Fraser J. Socioeconomic status, indoor and outdoor work, and malignant melanoma. Int J Cancer 1984; $34: 57-62.38$
24. Online Survey. http://swz.salary.com/salarywizard/layouthtmls/ swzl_co-mpresult_natio nal_HC07000221.html. Accessed 39 . September 2009

25. Elwood JM, Gallagher RP, Hill GB, Pearson JCG. Cutaneous melanoma in relation to intermittent and constant sun 40. exposure - the Western Canada Melanoma Study. Int J Cancer $1985 ; 35: 427-433$

26. Vagero D, Ringback G, Kiviranta H. Melanoma and other 41. tumours of the skin among office, other indoor and outdoor workers in Sweden 1961-1979. BrJ Cancer 1986; 53:507-512

27. Lew RA, Sober AJ, Cook N, Marvell R, Fitzpatrick TB. Sun exposure habits in patients with cutaneous melanoma: a case control study. J Dermatol Surg Oncol 1983; 9:981-986

28. Friedman RJ, Rigel DS, Silverman MK, Kopf A, Vossaert K. Malignant melanoma in the 1990's: the continued importance of early detection and the role of the physician examination and selfexamination of the skin. CA Cancer J Clin 1991; 41: 201-226 